\title{
Computed tomography of abdomen in staging and clinical management of lymphoma
}

\section{Summary and conclusions}

During July 1976 to December 1977, 150 patients with Hodgkin's disease and 138 with non-Hodgkin's lymphoma were examined by computed tomography (CT). In 45 cases 50 repeat examinations were conducted. Concurrent laparotomy and lymphography were performed on 68 and 56 patients respectively.

The overall incidence of false-positive CT examinations as confirmed by laparotomy was $7 \cdot 4 \%$. In 18 patients with non-Hodgkin's lymphoma in the abdomen there was good correlation between the two techniques. Of the 50 patients with Hodgkin's disease who underwent laparotomy, 17 had splenic disease and 14 minimally enlarged lymph nodes in 20 areas; CT, however, detected only four diseased spleens and five minimally enlarged lymph nodes. Nevertheless, CT often detected enlarged lymph nodes missed by lymphography and was $23 \%$ more efficient than lymphography in detecting unsuspected disease. CT also detected unsuspected disease in patients with relapse of lymphoma.

CT may replace other non-invasive investigations of abdominal disease in patients with lymphoma and give a reliable guide to prognosis. It does not, however, eliminate the need for laparotomy in staging Hodgkin's disease.

\section{Introduction}

Therapeutic regimens used to treat lymphoma depend on accurate assessment of sites affected by the disease, those in the abdomen being particularly difficult to evaluate. Clinical assessment has many shortcomings, and invasive procedures including laparotomy are required to stage the disease accurately. In many centres staging laparotomy is a standard investigation for apparently localised Hodgkin's disease.

Whole-body computed tomography (CT) is a non-invasive imaging technique for examining the abdomen and detecting enlarged lymph nodes. ${ }^{12}$ We report on a series of 288 patients with histologically confirmed Hodgkin's disease or non-

\footnotetext{
Department of Diagnostic Radiology, University of Manchester, Manchester M13 9PT

J J K BEST, MRCP, FRCR, senior lecturer in diagnostic radiology

W ST C FORBES, MB, FRCR, research fellow

I ISHERWOOD, MRCP, FRCR, professor of diagnostic radiology

CRC Department of Medical Oncology, University of Manchester, Christie Hospital and Holt Radium Institute, Withington, Manchester M20 9BX

G BLACKLEDGE, MB, MRCP, CRC research fellow

D CROWTHER, PHD, FRCP, CRC professor of medical oncology

Christie Hospital and Holt Radium Institute, Withington, Manchester M20 9BX

I D H TODD, MB, FRCR, consultant radiotherapist and chairman of Manchester Lymphoma Group

B EDDLESTON, MB, FRCR, consultant radiologist
}

Hodgkin's lymphoma who underwent CT either at presentation or during management.

\section{Patients and methods}

During July 1976 to December 1977, 288 patients included in the Manchester Lymphoma Group trials were referred from the Christie Hospital to the department of diagnostic radiology for CT scanning of the abdomen. Forty-five patients underwent repeat examinations, making a total of 338 separate scans. One hundred and ninety-one patients were scanned at presentation, 70 at the time of suspected relapse, and 27 during apparent complete remission. Altogether 177 patients were male and 111 female; their ages ranged from 10 to 73 years.

All patients had Hodgkin's disease or non-Hodgkin's lymphoma confirmed histologically, in most cases by lymph node biopsy but in the remainder from specimens of skin, liver, or bone. The histology was classified according to the Rye nomenclature for Hodgkin's disease and the Rappaport system for non-Hodgkin's lymphoma. ${ }^{3}$ There were 150 patients with Hodgkin's disease and 138 with non-Hodgkin's lymphoma, 59 of whom had the nodular or well-differentiated diffuse lymphocytic type and 79 diffuse lymphomas. At presentation, when the disease had been confirmed histologically, each patient was staged as accurately as possible according to the Ann Arbor classification..$^{5}$ In patients with Hodgkin's disease this followed the procedure described by Sutcliffe $e t \mathrm{al}^{\mathrm{l}}$ and included bipedal lymphography and laparotomy with splenectomy for patients with clinically localised disease. Similar investigations were carried out on patients with non-Hodgkin's lymphoma, except that all patients underwent bone marrow aspiration and trephine examination and laparotomy was not routinely performed. Eighteen of these patients, however, had already undergone diagnostic laparotomy.

CT at presentation was performed during investigation before treatment. In patients scanned during apparent remission or when relapse was suspected investigations appropriate to sites of suspected relapse were performed. All patients were scanned with an EMI CT5000 whole-body scanner, updated in July 1977 to the EMI CT5005. They were prepared for the examination with a bulk laxative for two days (Isogel, $10 \mathrm{ml}$ twice daily), and 30 minutes before scanning given $250 \mathrm{ml}$ Gastrografin by mouth diluted to $5 \% \mathrm{v} / \mathrm{v}$. Immediately before scanning the bowel was paralysed with an intramuscular injection of propantheline $30 \mathrm{mg}$. All patients were scanned supine, holding their breath in inspiration. Scanning was conducted at $140 \mathrm{kVp}$ and $28 \mathrm{~mA}$ at normal speed (20 seconds).

Patients with Hodgkin's disease were scanned from the level of the xiphisternum caudally. The first $8 \mathrm{~cm}$ was scanned at $1 \mathrm{~cm}$ intervals, and then at $2 \mathrm{~cm}$ intervals to the level of the superior anterior iliac spines. Scanning was continued caudally when there was a specific reason for doing so. Patients with non-Hodgkin's lymphoma were scanned from the level of the pulmonary hila to the superior anterior iliac spines at $2 \mathrm{~cm}$ intervals. The maximum skin entry dose was 5.3 rads. ${ }^{\text {? }}$

Scans were taken as positive when they showed enlargement of lymph nodes-that is, exceeding $1.5 \mathrm{~cm}$ in diameter. In some areas -namely, the retrocrural space and left para-aortic region-visible lymph nodes were regarded with suspicion even when smaller than this. The liver was considered to be enlarged when it appeared below the ribs in the mid-axillary line, and the spleen to be enlarged when it appeared below the ribs. These criteria were arbitrarily chosen as approaching those used clinically. Extranodal disease was diagnosed when the normal contour or architecture of the abdominal organs was distorted. Interpretation of scans was based on morphological criteria. Osseous disease required either loss of the normal bony outline with associated loss of fascial planes, implying adjacent soft-tissue infiltration, or attenuation increased above normal for bone. 
Contrast enhancement was used only when $(a)$ areas of low attenuation were seen in the liver and spleen, such areas being considered to be abnormal if they did not enhance after intravenous injection of contrast; and (b) renal disease and ureteric obstruction were suspected.

\section{Results}

The scans were examined retrospectively by JJKB and WStCF and compared with the lymphograms and results of laparotomy. CT was repeated in 45 patients to monitor progress and response to treatment.

\section{CT VERSUS LAPAROTOMY}

Staging laparotomies were performed on 50 patients with Hodgkin's disease (clinical stages I-IIIA); 21 disclosed evidence of disease (table). Twenty lymph node areas were affected in 14 patients, and in the remainder only the spleen was abnormal. In nine patients the scans suggested lymph node enlargement. Five of these patients had histological evidence of disease in the relevant nodes, whereas the remaining four had no disease detectable; these scans were therefore falsepositive. There was only one false-positive CT result in 29 patients with normal laparotomies. This was in the left para-aortic region.

Findings at laparotomy and on CT scanning in 50 patients with Hodgkin's disease: 21 operations yielded evidence of disease

\begin{tabular}{|c|c|c|c|c|c|}
\hline \multirow{2}{*}{ Sites of disease } & \multicolumn{2}{|c|}{ Laparotomy } & \multicolumn{3}{|c|}{ CT scan } \\
\hline & $\begin{array}{c}\text { No } \\
\text { disease }\end{array}$ & disease & Negative & Positive & $\begin{array}{c}\text { False- } \\
\text { positive }\end{array}$ \\
\hline $\begin{array}{l}\text { None seen at laparotomy.. } \\
\text { Spleen } \ldots . \\
\text { Coeliac nodes } \ldots\end{array}$ & 29 & $\begin{array}{r}17 \\
6 \\
4 \\
3 \\
3 \\
4\end{array}$ & $\begin{array}{r}28 \\
13 \\
4 \\
3 \\
3 \\
1 \\
3\end{array}$ & $\begin{array}{l}1 \\
4 \\
3 \\
3 \\
3 \\
3 \\
1\end{array}$ & $\begin{array}{l}1 \\
1 \\
2 \\
1\end{array}$ \\
\hline
\end{tabular}

CT failed to detect disease in 13 of the 17 abnormal spleens. In the remaining four cases the spleen was considerably enlarged (over three times normal weight). Nodular disease in the spleen was not detected. Only one patient had histological evidence of liver disease; in this case the liver was enlarged and seen to be so in the scan.

Eighteen patients with non-Hodgkin's lymphoma underwent laparotomy. This approach was different from that in Hodgkin's disease, since the laparotomies were diagnostic investigations and no elective operations were included. All patients presented with clinical evidence of intra-abdominal disease. In 14 cases CT detected disease in the major lymph node areas documented at laparotomy. CT understated the disease in three cases, and one did not cover the relevant area. Eleven out of $12 \mathrm{CT}$ examinations confirmed disease in the para-aortic area, and three out of five confirmed disease in the mesenteric region. There were no false-positive CT results in this group.

\section{CT VERSUS ABDOMINAL LYMPHOGRAPHY}

Lymphography was performed on eight patients with non-Hodgkin's lymphoma and 48 with Hodgkin's disease. Results were compared with CT for the area examined by lymphography-that is, the iliac and lower and mid-para-aortic lymph nodes. In 11 cases CT scans detected enlarged nodes in areas outside the lymphogram area. These included splenic hilar, upper para-aortic, retrocrural, and coeliac lymph nodes and one at the left renal hilum. The disease was confirmed histologically in seven cases, and in three out of four cases in which laparotomy was not performed lymph node enlargement seen in the scan regressed with treatment. This was shown by sequential CT examinations. In 47 cases the lymphograms were normal (43 patients with Hodgkin's disease, four with non-Hodgkin's lymphoma); CT scans were normal in 45 of these. In the remaining two cases enlarged nodes were detected by scanning in the lymphogram area. In both cases these were confirmed at laparotomy. Thirty-five other patients in this group had laparotomies confirming the absence of disease.
In nine patients with positive lymphograms CT also detected disease. Five of these patients underwent laparotomy, which in all cases confirmed the presence of disease. Two of the four remaining $w$ patients had follow-up examinations, which showed resolution of disease with treatment.

Nine of the 47 patients with normal lymphograms had evidence of intra-abdominal disease in the CT scan; in four of these disease was $\subseteq$ confirmed by follow-up scans. In an additional four patients with abnormal lymphograms, CT showed more disease than was indicated by lymphography and conventional staging procedures. Two of these patients had sequential CT scans, which showed regression of the disease visualised by scanning.

There were no false-negative CT scans of areas covered by lymphography, and in no case was disease understated.

\section{CT DURING RELAPSE}

Forty-four patients with Hodgkin's disease and 33 with nonHodgkin's lymphoma were scanned at the time of clinical or suspected relapse. In 16 patients with Hodgkin's disease there were signs of relapse only above the diaphragm. CT showed abdominal disease $\vec{v}$ in 14 of these. In all eight patients with suspected relapse CT showed enlarged lymph nodes in the abdomen. In 19 out of 20 patients $\vec{\sigma}$ with relapse detectable clinically in the abdomen scanning showed $v$ additional disease that was not suspected. Thirteen patients had scans at a later date which confirmed the response to treatment of the disease shown on scanning.

Of the 33 patients with relapse of non-Hodgkin's lymphoma, 28 had clinical evidence of intra-abdominal disease. Scanning showed $\mathbb{D}$

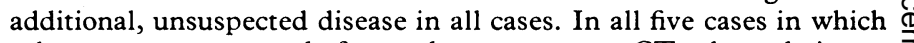
relapse was suspected from the symptoms CT showed intraabdominal disease. Twenty-three patients had scans at a later date to $\Phi$ monitor response to treatment.

\section{REMISSION ASSESSMENT}

Twenty-six patients with diffuse lymphoma (diffuse histiocytic lymphoma and diffuse poorly differentiated lymphocytic lymphoma) in apparent complete remission were scanned. Thirteen had normal scans, and 12 of these were alive 6-20 months later. Of the 13 patients with $\overrightarrow{\vec{B}}$ abnormal scans, only two were alive six and 20 months later $(P<0.01)$.

\section{Discussion}

This study on 288 patients with Hodgkin's disease and nonHodgkin's lymphoma is the largest published series of CT examinations in patients with lymphoma. ${ }^{8-15}$ The 68 laparoto- of mies and 56 lymphograms performed concurrently enabled the results of CT to be correlated with clinical, radiological, and $\frac{\text { ? }}{2}$ histological evidence of disease.

One aim of the study was to determine whether CT might $\mathrm{G}$ complement or replace staging laparotomy in the initial assess- $\frac{D}{0}$ ment of patients with Hodgkin's disease. In patients with intraabdominal manifestations the commonest site of disease is the 0

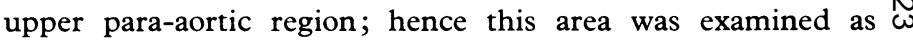
thoroughly as the technique allows. ${ }^{6}{ }^{16}$ Early experience in patients with non-Hodgkin's lymphoma, however, suggested 0 that the high retrocrural region was often affected, and the $\mathbb{\varnothing}$ examination was altered appropriately for these cases. The use + of bowel preparation enabled mesenteric lymph nodes and $T$ para-aortic nodes to be distinguished from loops of bowel in the scan.

Comparing the results of CT and laparotomy in the same $\stackrel{\mathbb{Q}}{\Omega}$ patient confirmed that CT may detect lymph node disease. The degree of confidence with which enlarged nodes are distinguished varies from site to site. In the para-aortic and retrocrural regions very small differences in size may be detected. ${ }^{17}$ In other areas where anatomy is more variable-for example, the mesenteric $\underset{\rightleftharpoons}{\rightleftharpoons}$ and coeliac regions - the degree of confidence in deciding whether nodes are enlarged is much less. Although qualitative differences in tissue density between normal and diseased nodes were 
sought, only abnormal size and shape were reliable indicators of disease.

In our series laparotomy in Hodgkin's disease was performed on patients with no clinical evidence of infradiaphragmatic disease and on a few patients (four) with clinical stage IIIA disease. Only minimal disease might therefore be expected within the abdomen. This group of patients showed the limitations of CT. Many small abdominal nodes (15 out of 20) were not detected, and the only false-positive scans in the series were in this group (table). One of the four false-positive nodes was in an area where nodes smaller than $1.5 \mathrm{~cm}$ in diameter carried a high index of suspicion (left para-aortic), and in all four cases the difference in size between the normal and reported abnormal nodes was small. In this study the overall incidence of falsepositive scans as confirmed by laparotomy was $7 \cdot 4 \%$ ( 5 out of 68 cases), which is reassuring.

CT cannot at present replace laparotomy because of its inability to detect abnormalities in the spleen. Infiltration by lymphoma in the spleen and liver may not be detected unless these organs are enlarged. The spleen, although often abnormal in Hodgkin's disease, is not usually grossly enlarged and may be of normal size. ${ }^{16}$ The inability of CT to detect disease in these organs reflects both the limitations of using the criterion of enlargement alone and the inherent limitations of the technique at present. CT, however, has definite advantages over lymphography. Lymphography examines relatively few abdominal lymph nodes. Within this region CT shows agreement with lymphography, and in our series improved on it. In addition, CT detects other disease, and we found a $23^{\circ}$ increase over lymphography in the detection of unsuspected abdominal disease. Such patients might therefore be spared laparotomy during staging investigations. CT may be repeated easily at any time after the first scan. Although the contrast medium used for lymphography may be seen for up to two years after examination, often there is insufficient contrast for adequate assessment of lymph node size within a few weeks or months, and with subsequent examinations lymphography becomes more difficult. ${ }^{18} \mathrm{CT}$ is therefore valuable for follow-up studies.

The ability of CT to aid in staging lymphoma according to the Ann Arbor classification is limited because of the low rate of detection of splenic disease and minimally enlarged lymph nodes. If, however, the aim of initial investigation is also to assess the extent of disease within the abdomen, CT provides an accurate, non-invasive means of visualising all abdominal lymph node areas.

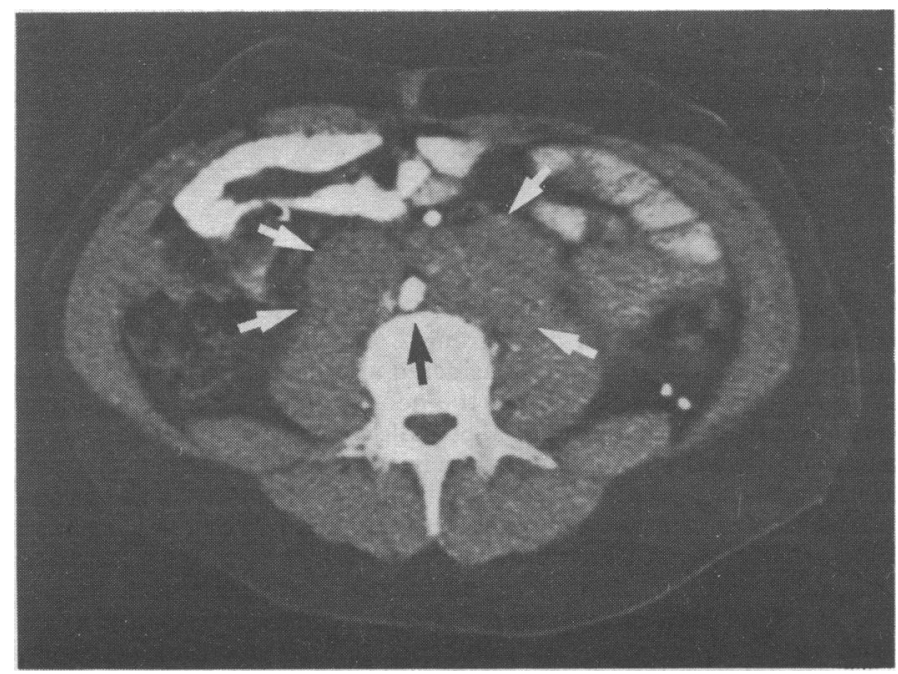

CT scan at L4 level taken after abdominal lymphography. Scan shows unopacified bilateral para-aortic lymph node masses that escaped detection by lymphography (white arrows) and single opacified lymph node (black arrow).
Examinations performed sequentially during management were extremely valuable. Residual disease after initial treatment or the full extent of relapsed disease may be accurately assessed. The response of disease to treatment as indicated by reduction in bulk may be monitored satisfactorily by CT, which in this respect is clearly superior to lymphography (see figure).

The unique ability of CT to detect occult abdominal disease in patients in clinical complete remission may help in predicting the course of the disease. An abnormal CT scan at this stage may prove to be an important prognostic indicator of survival in these patients and may indicate whether further treatment is necessary.

Although CT does not eliminate the need for laparotomy in staging Hodgkin's disease, its superiority over lymphography and ability to detect otherwise unsuspected disease in patients in relapse or remission make it an important investigation in the management of lymphoma. CT is a simple, non-invasive examination employing high technology, and the cost of an examination reflects the large initial cost and running expenses. Other investigations, however, such as lymphography and radioisotope scanning may be rendered unnecessary, and CT may then become the primary non-invasive investigation of abdominal disease in patients with lymphoma.

We thank the members of the Manchester Lymphoma Group for valuable co-operation in this work. The help of the secretarial staffs of the Christie Hospital and Holt Radium Institute and department of diagnostic radiology at the University of Manchester is also gratefully acknowledged. WStCF was funded by EMI Medical Ltd.

Requests for reprints should be addressed to: Dr J J K Best, Department of Diagnostic Radiology, University of Manchester, Stopford Building, Oxford Road, Manchester M13 9PT.

\section{References}

1 Kreel, L, Clinical Radiology, 1976, 27, 421.

2 Best, J J K, and Isherwood, I, Computerised Axial Tomography in Clinical Practice, p 411. Berlin, Springer-Verlag, 1977.

3 Lukes, R J, and Butler, J J, Cancer Research, 1966, 26, 1063.

${ }^{4}$ Rappaport, H, Atlas of Tumour Pathology, section 3, fasc 8. Washington DC, Armed Forces Institute of Pathology, 1966.

${ }^{5}$ Rosenberg, S A, Cancer Résearch, 1966, 26, 1225.

${ }^{6}$ Sutcliffe, S B J, et al, British Medical fournal, 1976, 2, 1343.

Isherwood, I, Neuroradiology. In press.

${ }^{8}$ Schaner, E G, et al, fournal of Computer Assisted Tomography, 1977, 1, 176.

${ }^{9}$ Redman, H C, et al, Radiology, 1977, 124, 381.

10 Alcorn, F S, et al, Radiology, 1977, 125, 717.

11 Schaner, E G, et al, Cancer Treatment Reports, 1977, 61, 1537.

12 Breiman, R S, et al, Radiology, 1978, 126, 159.

13 Jones, S E, Tobias, D A, and Waldman, R S, Cancer, 1978, 41, 480.

14 Pilepich, M V, et al, American fournal of Roentgenology, 1978, 131, 69.

15 Lee, J K T, et al, American fournal of Roentgenology, 1978, 131, 311.

16 Glatstein, E, et al, Radiology, 1970, 97, 425.

17 Callen, P W, Korobkin, M, and Isherwood, I, American fournal of Roentgenology, 1977, 129, 907.

18 Johnson, R G, and Cook, P L, American fournal of Roentgenology, 1968, 102,883 .

(Accepted 6 October 1978)

ONE HUNDRED YEARS AGO Dr J L Little reports a case where paregoric in small doses was administered to a child three weeks old for the relief of suffering caused by an inflammation of the knee-joint. The child gradually bore larger and larger doses; the paregoric was changed to tincture of opium, and this again to Magendie's solution. Soon the child obtained such a tolerance of this drug that, in a couple of months, from half a drachm to a drachm a day was necessary to quiet it. This state of things continued until the amount consumed by the child, less than eight months old, was two ounces of Magendie's solution in twenty-four hours. The dose was gradually diminished at the rate of about three drops a day, and, at the time of making the report, but ten drops were given at bedtime. The child's appearance improved very much; it was intelligent, and weighed eighteen pounds. (British Medical fournal, 1878.) 